\title{
Suppression of Trailing-Edge Noise Emitted by Two-Dimensional Airfoils
}

\author{
By Shohei TAKAGI ${ }^{1 * *}$ and Yasufumi KonISHI ${ }^{2) \dagger}$ \\ ${ }^{1)}$ Aerospace Research and Development Directorate, JAXA, Chofu, Japan \\ ${ }^{2)}$ National Agency of Vehicle Inspection, Kofu, Japan
}

(Received February 6th, 2009)

\begin{abstract}
Acoustic noise from the trailing edge of 2D airfoils is known to be discrete at certain moderate Reynolds numbers. The most widely accepted explanation for this phenomenon is the acoustic linkage between acoustic radiation and Tollmien-Schlichting (T-S) disturbances growing in the airfoil boundary layer. This paper has two objectives: (1) is to devise a technical method for suppressing or abating trailing-edge noise from a practical viewpoint; (2) is to obtain evidence whether or not T-S instabilities are associated with the frequency selection mechanism mentioned in the literature. From previous experiments (Atobe, T., et al., Trans. Jpn. Soc. Aeronaut. Space Sci., Vol. 52, pp. 74-80, 2009), generation of trailing-edge noise is dominated primarily by suddenly amplified unsteady disturbances in the presence of reverse flow on the pressure side of the airfoil rather than the suction side. Therefore, the boundary layer near the trailing edge on the pressure side is disturbed artificially with distributed roughness elements. As a result, the separation is swept away, and the trailing-edge noise is drastically suppressed. We reconfirmed that under the natural configuration with sound emission, most unsteady disturbances are not amplified by T-S instabilities, but rather by other inflectionaltype instabilities. The frequency selection mechanism in the tonal noise generation process remains unsolved.
\end{abstract}

Key Words: $\quad$ 2-D Airfoil, Trailing-Edge Noise, Boundary Layer Instability, K-H Instability

\section{Introduction}

Reduction of noise from aircraft is an urgent problem for people living near airports. Generally, there are two sources of aircraft noise: the engines, and the airframe itself. As higher-bypass-ratio engines have become more popular and aircraft have become larger, the amount of airframerelated noise in proportion to engine noise has grown. Airframe noise is associated with shear at the boundary layer and unsteady vortex shedding from landing gear, landinggear doors, and other separated flows as well as flap-edge flows. It makes significant contribution to the amount of acoustic energy, especially for large aircraft on approach.

Tonal noise emanates from landing gear and slats. Similar noise is emitted from the trailing edge of a 2D symmetrical airfoil for moderate Reynolds number flows. Generation of trailing edge (T-E) noise is generally explained as follows. $^{2-5)}$ First, the boundary layer is destabilized by Tollmien-Schlichting (T-S) instabilities, followed by abrupt growth of T-S waves in the reverse flow near the trailing edge on the pressure side of the airfoil with a some low angle of attack. When a T-S wave with a large amplitude is shed from the trailing edge, acoustic sound is generated. This acoustic sound spreads in the radial direction and is internalized somewhere in the flow on the airfoil. The reason why acoustic sound is discrete is ascribed to feedback loops between the T-E noise and T-S waves, although $\mathrm{T}-\mathrm{S}$ instability is inherently unstable with respect to broadband disturbances. However, this explanation does not

(C) 2010 The Japan Society for Aeronautical and Space Sciences

*Currently Muroran Institute of Technology, Muroran, Japan

${ }^{\dagger}$ Currently Tokyo Metropolitan University, Tokyo, Japan cover the case where T-S instabilities are subcritical at very low Reynolds numbers, as reported by Nakashima et al. ${ }^{6)}$ In addition, the experiments by Atobe et al. ${ }^{1)}$ and Kobayashi et al. ${ }^{7)}$ showed no T-S waves in generation of tonal noise, even for natural moderate Reynolds numbers. It appears that the mechanism of tonal noise generation from the trailing edge might be complex.

This paper aims to devise a method for suppressing or abating T-E noise as the first step in a project to solve the formidable problem of why T-E noise is monochromatic. For now, we focus on the separation region commonly observed near the trailing edge on the pressure side of the airfoil, where unstable disturbances are suddenly amplified. If separation can be artificially removed or weakened, T-E noise might be significantly reduced or suppressed. Based on this speculation, we attempted to experimentally manipulate the boundary layer near the trailing edge on the pressure side of an airfoil. The second purpose of this paper is to present experimental evidence about whether or not frequency selection is due to T-S instabilities.

\section{Experimental Setup and Procedures}

The experiment was conducted in the JAXA low-turbulence wind tunnel, with a rectangular test section span of $550 \mathrm{~mm}$ and a height of $650 \mathrm{~mm}$. Although the test section is not anechoic, sound reflection from the ceiling and floor is not crucial because the main purpose of the study is to suppress acoustic sound from the model. Both the ceiling and floor are slightly flared to remove the streamwise pressure gradient in the empty test section. The experimental setup is shown in Fig. 1. 


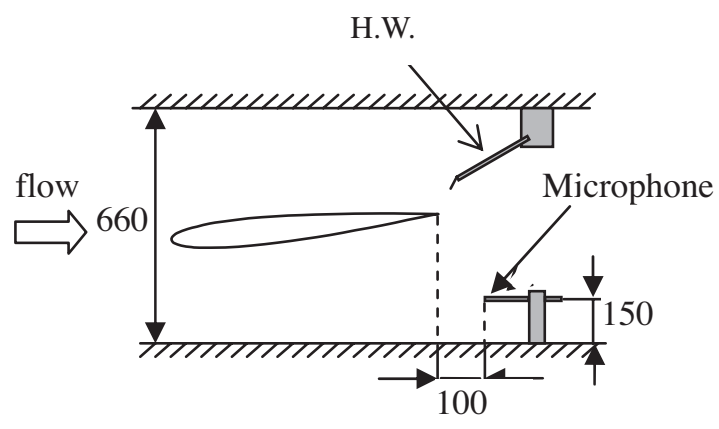

Fig. 1. Schematic of experimental setup with NACA0015 airfoil set at attack angle of -5 degrees, in-flow microphone and hot-wire probe on traversing mechanism. The flow is from left to right.

The free-stream velocity $U_{\infty}$ was set to $14.5 \mathrm{~m} / \mathrm{s}$. At this velocity, the free-stream turbulence level is less than $0.05 \%$ of the freestream velocity. The wing model has an NACA0015 cross-section with chord length and spanwise length of $400 \mathrm{~mm}$ and $550 \mathrm{~mm}$, respectively. The wing was set horizontally with an attack angle of -5 degrees so that the pressure side of the airfoil is the upper side, allowing the hot-wire probe on the $2 \mathrm{D}$ traversing mechanism from the ceiling to be surveyed readily on the pressure side. The streamwise velocity was measured with a constanttemperature hot-wire anemometer. The tungsten hot-wire sensor is $5 \mu \mathrm{m}$ in diameter and $1 \mathrm{~mm}$ in sensing length. The hot-wire probe was traversed in the streamwise $(x)$ and vertical $(y)$ directions.

The acoustic sound was measured with a microphone (B\&K type 4138), located $100 \mathrm{~mm}$ behind the trailing edge and $300 \mathrm{~mm}$ toward the suction side of the airfoil. A slitnose cone was attached ahead of the microphone with a blunt head shape to reduce self-induced acoustic noise. Time-dependent data for the velocity and acoustic noise were stored simultaneously on a PC via a 16-channel 16-bit A/D converter.

Previous experiments ${ }^{7)}$ showed that unsteady disturbances with T-E noise frequency are amplified abruptly in the separation region near the trailing edge, and then coherent acoustic sound is emitted when the amplified unsteady disturbances are shed from the trailing edge. Therefore, acoustic sound might be weakened or suppressed if the separation structure can be destroyed or removed by some device. In the present experiment, isolated roughness elements $3 \mathrm{~mm}$ in diameter and $4 \mathrm{~mm}$ in height were distributed in double rows at $17.5 \mathrm{~mm}$ spanwise intervals in a staggered formation at a streamwise distance of $5 \mathrm{~mm}$ at $92.5 \%$ of the chord length on the pressure side.

\section{Results and Discussion}

The basic characteristics of the acoustic sound emitted from the trailing edge of the NACA0015 airfoil were measured as a function of uniform velocity. The results are shown in Figs. 2 and 3. Figure 2 shows the typical frequency spectrum of the sound pressure as captured by the in-flow

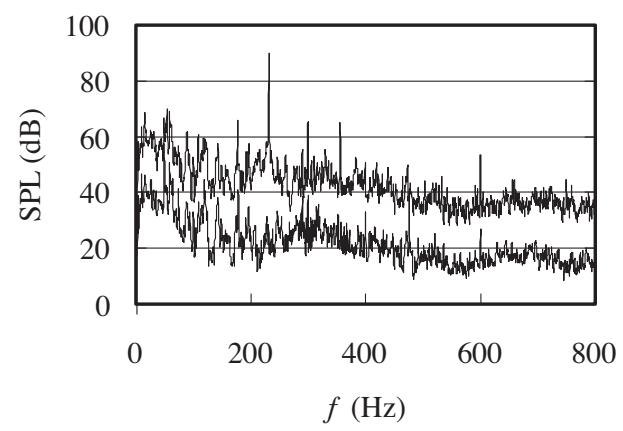

Fig. 2. Sound spectrum obtained from in-flow microphone together with background noise spectrum plotting $-20 \mathrm{~dB}$ shift at uniform velocity of $14.5 \mathrm{~m} / \mathrm{s}$.

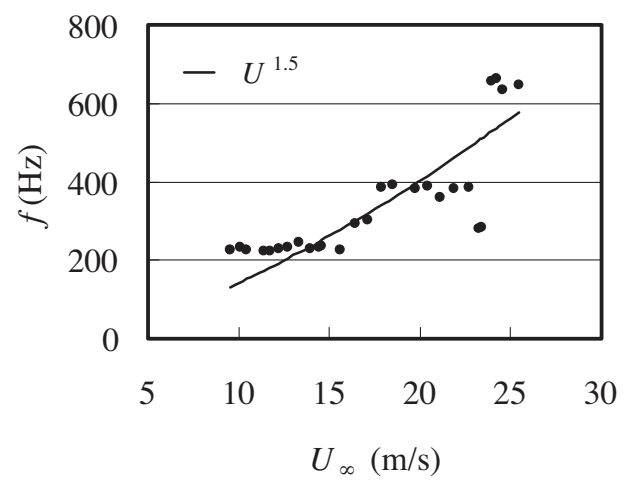

Fig. 3. Sound frequency as function of uniform velocity.

Solid line represents $f=0.011 U^{1.5} / \sqrt{\nu C}$ (Paterson $^{3)}$ ), where $v$ and $C$ are the kinematic viscosity and the chord length of the model, respectively.

microphone at a free stream velocity of $14.5 \mathrm{~m} / \mathrm{s}$. In this figure, the background noise spectrum in the empty test section is also superimposed with a plot of the $-20 \mathrm{~dB}$ shift. In comparison to both spectra, the discrete sound higher than $30 \mathrm{~dB}$ above the background level peaks sharply at $232 \mathrm{~Hz}$. Figure 3 shows frequency dependence of the tonal noise on the uniform velocity. The general characteristics of tonal noise are obtained from the empirical relation $f=$ $0.011 U^{1.5} / \sqrt{\nu C}$ proposed by Paterson. ${ }^{3)}$ This relation comes from the fact that the Strouhal number is a constant based on the uniform velocity and the laminar thickness of the boundary layer as the characteristic length. In other words, the characteristic length for the Strouhal number is a function of the model chord length rather than the model thickness. This is different from the Strouhal number of 2D bluff bodies. Figure 3 also shows that there is a ladder-like structure with a constant slope on the general trend curve, which has been found by numerous researchers ${ }^{1-3)}$ in conventional closed test sections to be prone to sound reflections from hard walls, resulting in acoustic resonance. Contrarily, Nash et al. ${ }^{8)}$ demonstrated that each step does fit the $U^{0.8}$ dependence in anechoic environments. Since the overall trend coincides with previous observations with and without acoustic resonance, we do not discuss this point further, and attempt to suppress generation of tonal noise.

Figure 4(a) shows the disturbance growth against the streamwise direction on the pressure side, where $u_{\mathrm{f}}^{\prime}$ and $u^{\prime}$ 

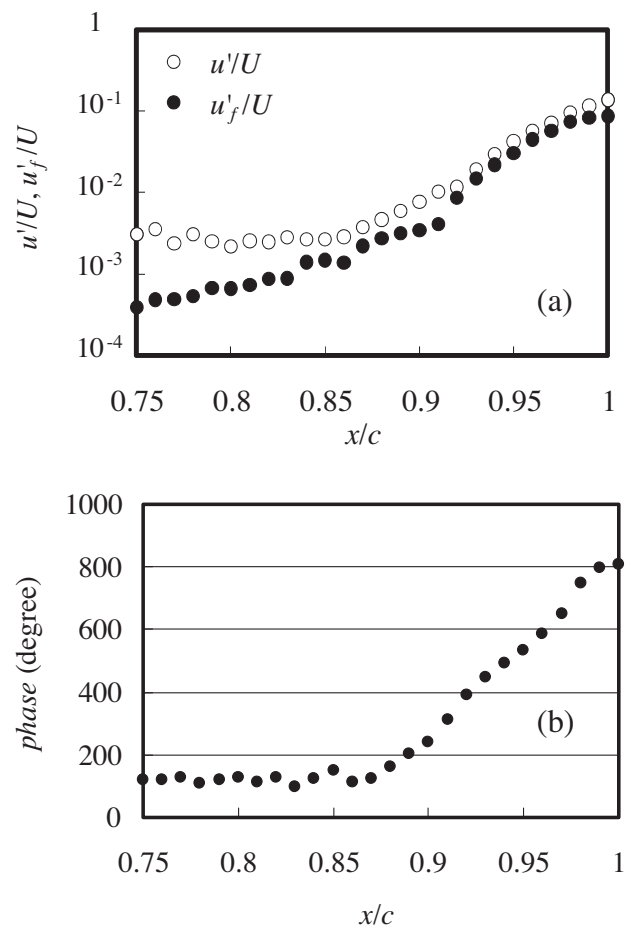

Fig. 4. (a) Disturbance development and (b) phase distribution in chordwise direction on pressure side. Phase shift is measured with the signal from the microphone used as a reference.

denote the RMS value of the discrete frequency component and the overall RMS value of the velocity fluctuations, respectively. We can ignore that the discrete frequency in the $u$ component coinciding with the tonal noise frequency in the sound pressure. As seen from the overall RMS value, the disturbances start to grow exponentially downstream of $x / c=0.875$. The discrete frequency component and its higher harmonics contribute to the exponential growth. Therefore, the flow is highly periodic. Since the magnitude of the overall disturbance exceeds $10 \%$ of the free stream velocity $U_{\infty}$ near the airfoil surface, a significantly strong vortex is shed from the trailing edge on the pressure side.

Figure 4(b) shows the phase distribution of the velocity fluctuations detected by the hot-wire sensor in the chordwise direction on the pressure side. Phase shift is measured with the signal from the microphone used as a reference. Although the hot-wire sensor is generally sensitive to sound, its effect was ignored because the hot-wire sensor is usually less sensitive to sound than to velocity. Therefore, Fig. 4(b) indicates that coherent velocity fluctuations with acoustic sound frequencies obviously propagate downstream at a constant phase velocity downstream of $x / c=0.875$. On the other hand, no change in phase is observed upstream of $x / c=0.875$, indicating that the discrete component with intensity smaller than $0.1 \%$ with respect to $U_{\infty}$ is not due to velocity fluctuations, but is sound pressure generated at the trailing edge. The phase velocity of the amplifying velocity fluctuation downstream of $x / c=0.875$ is about $40 \%$ of the uniform velocity $U_{\infty}$, which is deduced from the discrete frequency and the wavelength of one cycle from the gradient
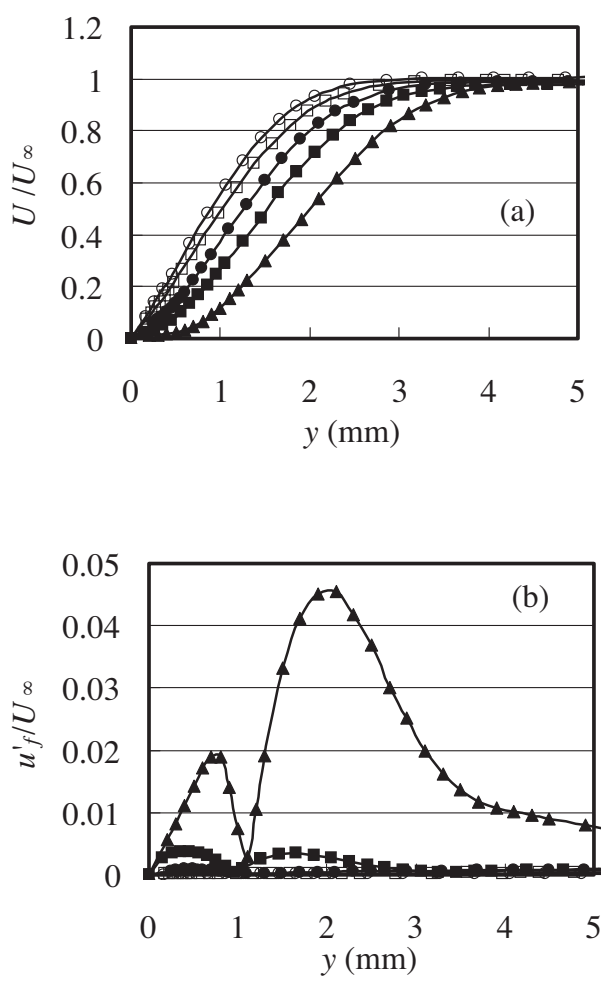

Fig. 5. Distributions of (a) mean velocity and (b) RMS values of the, T-E noise component. $\bigcirc, x / c=0.75 ; \square, x / c=0.8 ; \quad \bigcirc, x / c=0.85$; $\boldsymbol{\square}, x / c=0.9 ; \boldsymbol{\Delta}, x / c=0.95$.

in Fig. 4(b). Note that this phase velocity is close to that of T-S waves in the boundary layer developing along the airfoil. $^{8)}$

Next, we examined why the velocity fluctuations are amplified abruptly near the trailing edge. The mean velocity and RMS value of the tonal noise component in the $y$ direction at various locations in the $x$ direction around $x / c=0.875$ on the pressure side were measured as shown in Figs. 5(a) and (b). As $x$ increases, the velocity profile near the airfoil surface becomes more inflectional, corresponding to the growth of the T-E noise component. At $x / c=0.95$, the boundary layer is just about to separate.

Thus, the inflectional velocity profile is unstable, resulting in rapid growth of unsteady disturbances as noted in Fig. 4. Further downstream of $x / c=0.95$, there is reverse flow near the airfoil surface. As shown in Fig. 5(b), the intensity distributions of the T-E noise component have double peaks. The major peak is located at the inflection point on the profile and in minor places inside the reverse flow region.

Numerous studies have reported that the reverse flow, which is related with the separation formed near the trailing edge region on the pressure side of the airfoil, plays an important role in generation of tonal noise.

This is why unsteady disturbances are abruptly magnified in the separation region. Therefore, if the separation can be prevented by some method, tonal noise might be suppressed. For the present experiment, isolated roughness elements with a diameter of $3 \mathrm{~mm}$ and a height of $4 \mathrm{~mm}$ were distrib- 


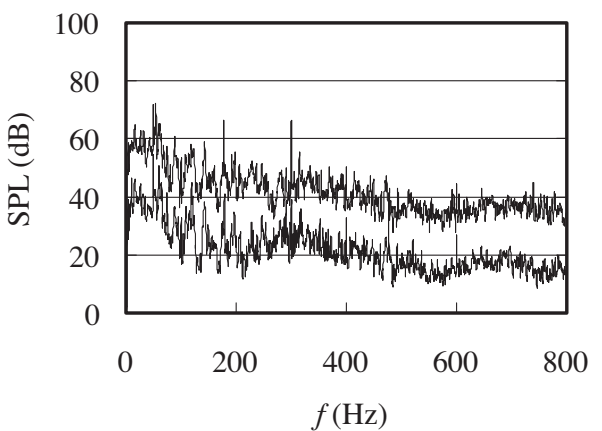

Fig. 6. Frequency spectrum of sound pressure when flow condition artificially controlled using isolated roughness elements plotted with background noise spectrum $-20 \mathrm{~dB}$ shift at uniform velocity of $14.5 \mathrm{~m} / \mathrm{s}$.

uted in staggered formation at $92.5 \%$ of the chord length on the pressure side. The sound-pressure spectral analysis result is shown in Fig. 6. Once the boundary layer on the pressure side is artificially disturbed, the tonal noise disappears completely, suggesting that roughness elements destroy the separation structure and prevent shedding of the $2 \mathrm{D}$ coherent vortex, causing the strong discrete acoustic emission.

Figure 7 shows the distribution of the mean velocity, the overall RMS value of the velocity fluctuation and the phase velocity at $x / c=0.9$ with and without roughness elements, which are distributed at $x / c=0.925$. The frequency spectra of the $u$-component at $x / c=0.9$ with and without roughness elements are compared in Fig. 8.

The roughness elements exerted no visible effect on the mean velocity distribution, although the flow downstream of the roughness elements is drastically changed from laminar to turbulent state, which is not shown here. This suggests that the inflection on the mean velocity profile is not due to direct influence from the vortex shedding motion but due to an unfavorable pressure gradient near the trailing edge. On the other hand, the overall RMS profile is definitely altered from two peaks to a single peak, as shown in Fig. 7(b), indicating that the two peaks are mainly due to the contribution of the velocity fluctuation to the tonal noise frequency, as shown in Fig. 8. This comparative analysis shows that the acoustic disturbance is internalized into the boundary layer just around $x / c=0.85$, as shown in Fig. 4(a), and then the internalized disturbance starts to grow exponentially.

It is shown by virtue of suppression of the T-E noise that there is obviously an acoustic loop between the $u$ component and the T-E noise originating from the vortex shedding. However, one important problem which remains unsolved is how the frequency of the tonal noise is selected. It should be noted that the phase distribution shown in Fig. 7(c) with respect to the amplifying velocity fluctuation does not resemble the T-S wave observed in the $2 \mathrm{D}$ boundary layers, where there is an abrupt phase change at 180 degrees at the outer edge within the boundary layer, which characterizes the T-S instability wave as shown by a theoretical approach. ${ }^{9)}$ This incongruity suggests that, as already
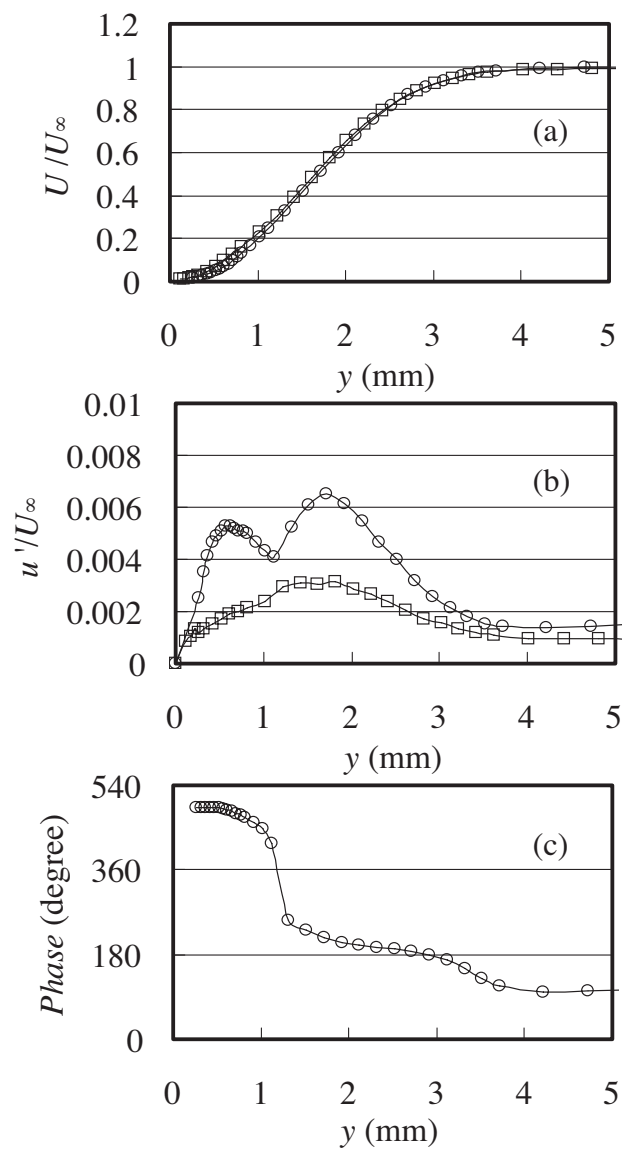

Fig. 7. Distributions of (a) mean velocity, (b) and (c) overall RMS values and phase angles of fluctuating velocity with reference to microphone signal at $x / c=0.9$ just upstream of distributed roughness.

$\bigcirc$, natural case; $\square$, artificially disturbed case.

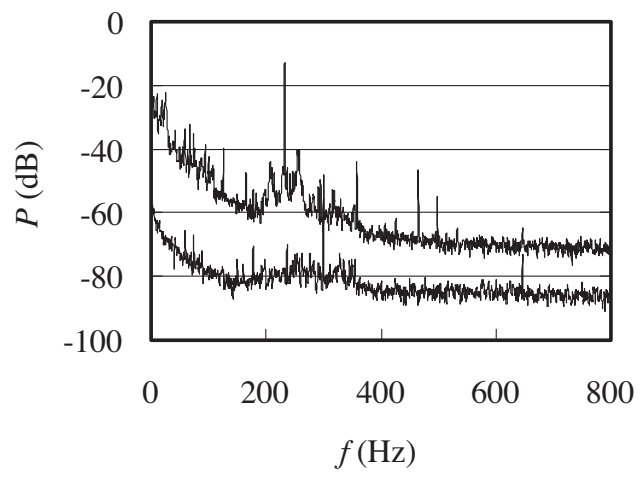

Fig. 8. Frequency spectra of velocity fluctuations at $x / c=0.9$.

The top spectrum is the natural case and the bottom with $-20 \mathrm{~dB}$ shift is the artificially disturbed case.

shown in Fig. 8, the moderate peak consisting of broadband disturbances peaking at about $270 \mathrm{~Hz}$, which is very close to the frequency of the tonal noise, is obviously not attributable to T-S instabilities. This is consistent with our previous observations, ${ }^{1)}$ which concluded that the broadband disturbances originate from hydrodynamic instability of the inflectional velocity profile and as a consequence, visualized flow motions show that unsteady disturbances seem to be sequentially magnified during a half period, after the turbulent boundary layer is shed from the suction side of airfoil. 
In this sequence, the flow near the wall at the pressure side near the trailing edge moves upstream and the boundary layer far above the wall there flows forward. Therefore, our previous work suggests that this flow system generating a high-shear layer with an inflection point at some boundary layer position gives the flow condition for the KelvinHelmholtz instability. However, it seems we cannot conclude that the K-H instability is related directly to the frequency selection of the T-E noise, because the nondimensional frequency of the T-E noise is about 0.056 for Strouhal number based on the uniform velocity and the boundary layer thickness at $x / c=0.9$ shown in Fig. 7. Similar inflectional instability, the so-called secondary instability or highshear-layer instability, is observed in the late stage of both $2 \mathrm{D}$ and 3D boundary layer transition processes prior to turbulent state. According to Nishioka et al. ${ }^{10)}$ for $2 \mathrm{D}$ parallel channel flow, the Strouhal number is 0.36 , based on the half width of channel and the center velocity. For 3D cases, Takagi et al. ${ }^{11)}$ and Kawakami et al. ${ }^{12)}$ observed a Strouhal number of 0.35 in a yawed-cylinder boundary layer and 0.34 in a flat-plate boundary layer with a swept imposed body above the plate. Surprisingly, the Strouhal number independent of $2 \mathrm{D}$ and $3 \mathrm{D}$ boundary layers is approximately 0.35 , which is inconsistent with the present hydrodynamic instability. This incongruity suggests that the present inflectional instability near the trailing edge may not be associated directly with the frequency selection mechanism of the trailing-edge noise. This observation also contrasts with the result that $\mathrm{T}-\mathrm{S}$ instabilities play an important role in the frequency selection mechanism observed by Nash et al. ${ }^{8)}$ and others. To unravel the frequency-selection mechanism, we need further investigation of the interaction between the inflectional boundary layer near the trailing edge and the wake of the airfoil, where a street of Karman vortices is formed.

There is another difference in the spectra where components with frequencies lower than that of the discrete $u$ component are attenuated when roughness elements are used. This indicates that the mean flow of the boundary layer with no roughness elements fluctuates at a low frequency associated with the fact that the separation point on the pressure side is unsteady.

\section{Conclusion}

We tried to devise a method for suppressing or abating tonal noise from the trailing edge of a 2D NACA0015 airfoil at an angle of attack of -5 degrees at moderate Reynolds numbers. We tried to clarify frequency selection mechanism of this trailing-edge noise. With respect to these two goals, we focused on the relation between tonal noise and flow separation near the trailing edge.

Distributed roughness elements designed to remove the separation were attached to the airfoil near its trailing edge, resulting in successful suppression of sound emission because the flow at the boundary layer near the trailing edge became turbulent, and reverse flow was removed. With no roughness elements, the phase velocity measurements of the abruptly growing disturbance in the reverse flow region showed that the disturbance does not originate from T-S instabilities, but from other inflection-type instabilities, which contrasts with common opinion. The frequency selection mechanism in tonal noise generation remains unsolved.

\section{References}

1) Atobe, T., Tuistra, M. and Takagi, S.: A Study on Airfoil Tonal Noise Generation in Resonant Environments, Trans. Jpn. Soc. Aeronaut. Space Sci., 52 (2009), pp. 74-80.

2) Clark, L. T.: The Radiation of Sound from an Airfoil Immersed in a Laminar Flow, Trans. ASME A: J. Eng. Power, 93 (1971), pp. 366-376.

3) Paterson, R. W., Vogt, P., Fink, M. R. and Munch, C. L.: Vortex Noise of Isolated Airfoils, J. Aircraft, 10 (1973), pp. 296-302.

4) Tam, C. K. W.: Discrete Tones of Isolated Airfoils, J. Acoust. Soc. Am., 55 (1974), pp. 1173-1177.

5) Akishita, S.: Tone-like Noise from an Isolated Two Dimensional Aerofoil, AIAA Paper 86-1947, 1986.

6) Nakashima, A. and Akishita, S.: Discrete Tone Noise on TwoDimensional Wing (Reconstruction of Time Sequential Flow Fluctuation Pattern), Trans. Jpn. Soc. Mech. Eng. B, 61 (1995), pp. 2115-2120 (in Japanese).

7) Kobayashi, H., Nishizawa, A. and Takagi, S.: Effects of a 2-D Roughness on Tonal Noise Generated by an NACA0012 Airfoil, J. Jpn. Soc. Aeronaut. Space Sci., 55 (2007) pp. 527-532 (in Japanese).

8) Nash, E. C., Lowson, M. V. and McAlpine, A.: Boundary Layer Instability Noise on Airfoils, J. Fluid Mech., 382 (1999), pp. 27-61.

9) Itoh, N.: The Progress in Fluid Dynamics-Boundary Layers-, Tani, I., ed., Maruzen Co., Ltd., Tokyo, 1984, p. 104 (in Japanese).

10) Nishioka, M., Asai, M. and Iida, S.: Experimental Study on Secondary Instability, Proc. Turbulence Symposium, 1979, pp. 53-60 (in Japanese).

11) Takagi, S., Tokugawa, N. and Itoh, N.: Turbulent Transition from an Point Source in 3-D Boundary Layer on Yawed Circular Cylinder, Proc. Turbulence Symposium, 1997, pp. 115-116 (in Japanese).

12) Kawakami, M., Kohama, Y. and Okutsu, M.: Stability Characteristics of Stationary Crossflow Vortices in Three-Dimensional Boundary Layer, AIAA Paper 99-0811, 1999. 